\title{
Cathode lens spectromicroscopy: methodology and applications
}

\author{
T. O. Menteş ${ }^{* 1}$, G. Zamborlini ${ }^{2,3}$, A. Sala ${ }^{1}$ and A. Locatelli ${ }^{1}$
}

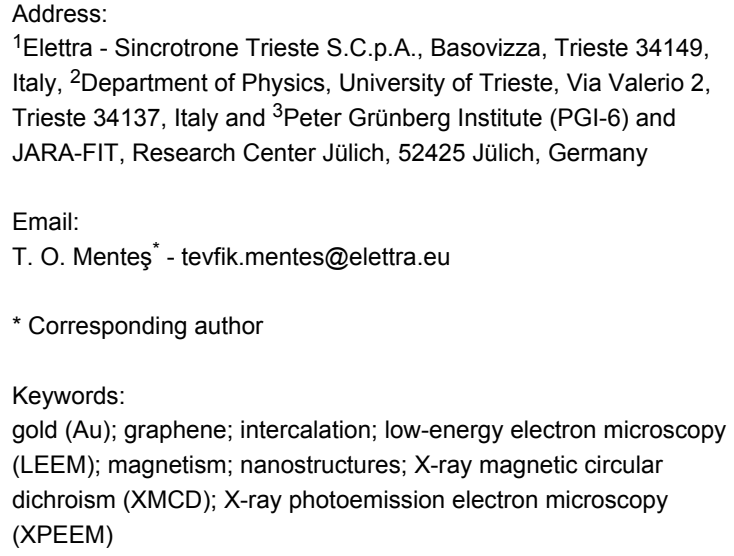

Beilstein J. Nanotechnol. 2014, 5, 1873-1886.

doi:10.3762/bjnano.5.198

Received: 09 July 2014

Accepted: 25 September 2014

Published: 27 October 2014

This article is part of the Thematic Series "Atomic scale interface design and characterisation: Experimental aspects and methods".

Guest Editor: C. Bittencourt

(C) 2014 Menteş et al; licensee Beilstein-Institut. License and terms: see end of document.

\begin{abstract}
The implementation of imaging techniques with low-energy electrons at synchrotron laboratories allowed for significant advancement in the field of spectromicroscopy. The spectroscopic photoemission and low energy electron microscope, SPELEEM, is a notable example. We summarize the multitechnique capabilities of the SPELEEM instrument, reporting on the instrumental aspects and the latest developments on the technical side. We briefly review applications, which are grouped into two main scientific fields. The first one covers different aspects of graphene physics. In particular, we highlight the recent work on graphene/Ir(100). Here, SPELEEM was employed to monitor the changes in the electronic structure that occur for different film morphologies and during the intercalation of $\mathrm{Au}$. The Au monolayer, which creeps under graphene from the film edges, efficiently decouples the graphene from the substrate lowering the Dirac energy from $0.42 \mathrm{eV}$ to $0.1 \mathrm{eV}$. The second field combines magnetism studies at the mesoscopic length scale with self-organized systems featuring ordered nanostructures. This example highlights the possibility to monitor growth processes in real time and combine chemical characterization with X-ray magnetic circular dichroism-photoemission electron microscopy (XMCD-PEEM) magnetic imaging by using the variable photon polarization and energy available at the synchrotron source.
\end{abstract}

\section{Introduction}

The cathode lens, or immersion objective lens, is used to image electrons emitted from surfaces [1]. In a microscope that uses this type of objective, the sample surface acts as the cathode held at a negative potential, whereas the anode (objective lens) has a central aperture to allow for the passage of the emitted electrons towards the imaging column. The imaged electrons 
may originate from different processes such as thermionic emission, secondary emission, emission of photoelectrons from core levels and the valence band or elastic backscattering [2] Methods based on the latter two, photoemission electron microscopy (PEEM) and low energy electron microscopy (LEEM), have found a special place in the field of surface science, and they will be the focus of our review.

During the evolution of PEEM [3], its first use as an X-ray microscope in a synchrotron environment in the late eighties stands out as one of the most significant developments [4]. Since then X-ray PEEM (XPEEM) has become a widespread analytical technique for surface investigation, which takes advantage of the high photon flux along with the tunable energy and polarization available at synchrotron sources [5]. In recent years, the natural combination of XPEEM with LEEM has created the powerful surface science facility, spectroscopic photoemission and low energy electron microscope (SPELEEM). In SPELEEM, the structural sensitivity of LEEM perfectly complements the chemical and magnetic information provided by XPEEM, thus creating a complete characterization tool of material properties at the nanometer length scale.

The following provides an overview on SPELEEM methods along with the recent examples predominantly considering the activity carried out at the Elettra Sincrotrone Trieste. The first part of the paper is organized as an extended introduction to LEEM and XPEEM methodology. Then, we give a detailed account of the instrumental aspects specific to the SPELEEM instrument at Elettra.

The bulk of this work is dedicated to applications of the SPELEEM technique. We put special emphasis on graphene, which has been extensively studied by using cathode lens microscopy, LEEM in particular, with numerous studies of epitaxial graphene grown on a variety of transition metal and silicon carbide supports. These microscopy experiments have been carried out by using well-established methodologies, which were formerly developed for the analysis of ultra-thin metal films on single crystal surfaces [6]. These methods were soon adapted to the needs of the rising research field of graphene, making LEEM one of the prominent methods to access the structural properties of graphene [7]. Since several review works have already addressed this subject [8-10], treating in depth also the experimental methods, the section on graphene is limited to an overview on the most recent research activity. The versatility of the LEEM and SPELEEM methodologies will be further illustrated by the effect of Au intercalation in graphene on $\operatorname{Ir}(100)$. The last part of the paper focuses on the studies of magnetism at the nanoscale using the SPELEEM.

\section{Review}

\section{Low energy electron microscopy}

Low energy electron microscopy (LEEM) is a surface-sensitive method based on the elastic backscattering of low energy electrons $[6,11]$. The concept was put forth by Ernst Bauer in the 1960 s, and the first operating instrument was demonstrated by Telieps and Bauer [12]. "Low energy" stands for electron energies from a few to several hundred electronvolts. Importantly, due to the high elastic backscattering cross section in the very low energy range (2-20 eV), exposure times are short and data collection becomes possible at video frame rate in most cases. Figure 1a and Figure 1b provide a schematic diagram of typical LEEM and PEEM instrumentation. Backscattered electrons are collected by the objective lens (also known as cathode lens or immersion lens), of which the sample is part. The objective lens, which is the most important optical element of the microscope, accelerates the e-beam to an energy of several $\mathrm{keV}$. The outgoing beam is manipulated by a dedicated set of electron optical elements in the imaging column, which produces a magnified image of the sample. In order to combine the low energy scattering and the high energy imaging stages, a high voltage bias between the sample and the objective lens acts as a decelerating/accelerating potential for the incident/scattered electrons. Besides the electron energy, the backscattering geom-

a)

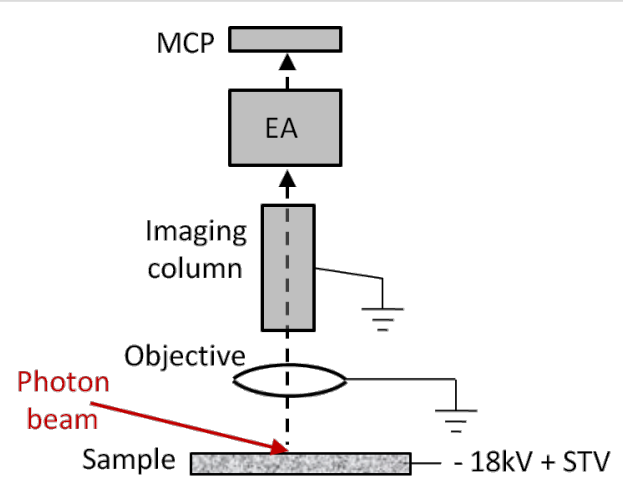

b)

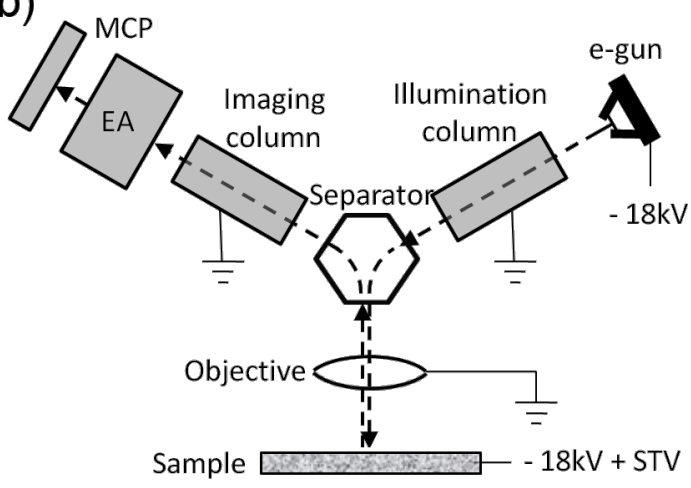

Figure 1: The simplified schematic description of a) XPEEM, b) LEEM The energy analyzer (EA) is optional in both cases. Panel (b) with the energy analyzer represents also the SPELEEM setup. 
etry at normal incidence distinguishes LEEM from other more conventional electron microscopies. This necessitates a beam separator, which is used to separate the incident and the scattered electron beams [6].

Contrast mechanism. Among all contrast mechanisms available in LEEM, "diffraction contrast" is the one that is most commonly used. This is derived from the strong energy dependence of electron diffraction intensities, making LEEM suitable for studying crystalline systems [13]. The backscattering intensity varies depending on the material, presence of adsorbates, formation of surface reconstructions and other ordered structures, giving the means to distinguish lateral variations in such properties. In the basic operation mode, only one of the low energy electron diffraction (LEED) beams is used to produce an image, in which the energy-dependent intensity provides information about the local morphology and crystal structure. This is done by filtering out undesired diffraction beams in the backfocal plane of the objective lens by using an aperture (usually called contrast aperture). The selection of the specular beam (zero-order diffraction) is commonly referred to as the bright field mode. An illustration of the intensity variations resulting from diffraction contrast is shown in Figure 2. The three curves belong to clean $\mathrm{W}(110)$, to $\mathrm{W}(110)$ covered with a pseudomorphic Fe monolayer, and to $\mathrm{O}(1 \times 12) / \mathrm{W}(110)$. As seen in the top panels, the first two surfaces give the same $(1 \times 1)$ LEED pattern, whereas the oxygen-covered surface features an additional superstructure. Nevertheless, all LEEM $I(V)$ curves show distinct differences. Similar differences are observed on surfaces with different structure and composition, which produces a contrast between regions of laterally-varying morphology by appropriate choice of the electron energy.

The sharp change in intensity at very low energy, seen in the inset of Figure 2, corresponds to the transition into the so-called mirror electron microscopy (MEM) regime. This MEM-LEEM transition marks the onset for the total reflection of incident electrons as the electron energy is lowered. The threshold energy predominantly depends on the surface work function and on the angle of incidence of the electrons. Therefore, imaging at or near the MEM transition allows to map the local work function as well as the variations in the surface topography. The effect of the work function is clear in the inset of Figure 2, in which the adsorption of oxygen on W(110) results in a work function more than $1.2 \mathrm{eV}$ higher than that of the clean surface, with a corresponding shift in the MEM-LEEM transition. Fe adsorption, instead, induces a less pronounced shift towards a lower work function.

The diffraction contrast is also exploited in the dark field mode, obtained by imaging with higher order LEED beams. The

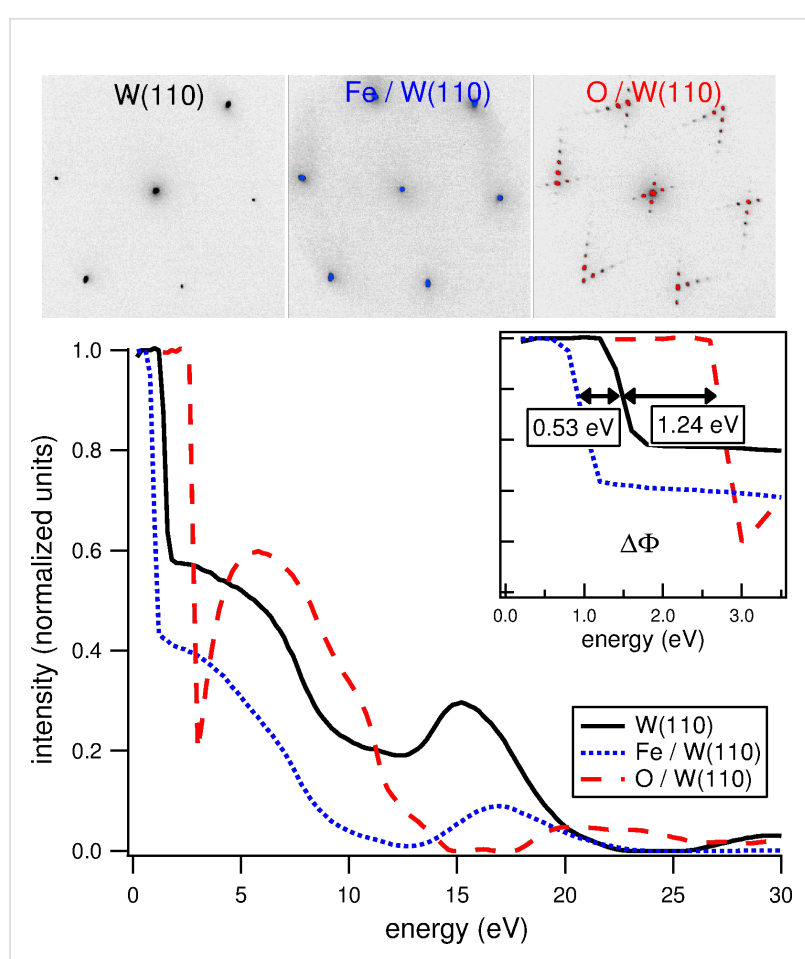

Figure 2: Energy dependence of the $(00)$ beam intensity for clean, Fe-covered and O-covered W(110) surfaces. The top panels show the respective LEED patterns. The inset is a blowup of the MEM-LEEM transition at low energy. The increase (decrease) in the work function due to the presence of $\mathrm{O}$ or $\mathrm{Fe}$ is seen in the shift of the transition energy.

diffraction order is selected by placing the contrast aperture on the desired beam. The resulting real space image gives a direct map of the corresponding structure. No intensity is seen elsewhere, except that originating from the diffuse background of the primary diffracted beam. The lateral resolution is comparable to that of the bright field mode, and the acquisition times, although slightly longer than the bright field operation, can be a few seconds to minutes depending on the intensity in the selected diffraction order.

Due to the short inelastic mean free path (IMFP) at low electron energies below a few hundred electronvolts [14], LEEM is a surface sensitive technique, which probes only a few atomic layers near the surface. Nevertheless, below $10 \mathrm{eV}$ the IMFP considerably increases (up to a few nanometers) giving depth information to LEEM. This is best reflected in the quantum size oscillations in electron reflectivity for thin films on crystalline surfaces $[11,15]$. As regularly observed in LEEM, the period of intensity oscillations as a function of the electron energy is highly dependent on the film thickness. Beyond their period, the amplitude of these quantum size oscillations depends on the film thickness and the IMFP, which was recently used to extract the IMFP in metal films at very low electron energies [16]. 
$\boldsymbol{\mu}$-LEED operation mode is a natural extension of LEEM. For crystalline surfaces, the backfocal plane of the objective lens contains the diffraction pattern from the probed area, which can be transferred to the detector with the proper lens excitation in the imaging column. By placing a small aperture in the illumination column or at the image plane of the objective lens, the probed area can be limited to a micrometer-sized region, thus giving rise to the micro-probe operation. When the length scale of the structural heterogeneity is below the size of the microprobe, the contribution of different domains to the LEED pattern can still be sorted out by using LEED in combination with dark-field LEEM imaging. The micro-probe capability is especially useful in quantitative structure analyses of LEED $I(V)$ curves acquired from single domains on a heterogeneous surface. The first example of a full surface structural analysis at the micrometer scale by using LEED $I(V)$ in a LEEM instrument was given only recently for the case of the $(4 \times 4)$ reconstruction of oxygen on $\operatorname{Ag}(111)$ [17].

Beyond the laterally-resolved electron diffraction, LEED measurements in a LEEM instrument have practical advantages such as electron-energy independent spot positions and constant electron flux. The former is due to the acceleration stage at the objective lens, after which the electrons reach $18 \mathrm{keV}$ regardless of the start energy (i.e., the energy of the elastically-scattered electrons at the sample surface). It should be underlined that this is particularly useful in the analysis of energy-dependent $I(V)$ data.

\section{X-ray photoemission electron microscopy}

PEEM uses UV or soft X-ray photons to stimulate the emission of photoelectrons to probe the state of the emitter. A simplified sketch of an XPEEM setup is given in Figure 1a. Similar to LEEM, it is based on the cathode lens, which accelerates the photoelectrons to an energy of several $\mathrm{keV}$ and directs them towards the imaging column of the instrument. The low photon energy of the conventional photon sources readily available in most laboratories presents a limitation, as the information on surface chemistry is available in core-level electronic transitions, which are only accessible by using higher photon energies from few tens of electronvolts to above $1 \mathrm{keV}$. By providing tunable high-brightness X-ray beams, synchrotron sources greatly extend the application field of XPEEM instruments, which can achieve chemical, magnetic and electronic structure contrast through the implementation of the most popular photoelectron spectroscopies such as X-ray absorption spectroscopy (XAS), photoelectron spectroscopy (XPS), and angle-resolved photoemission spectroscopy (ARPES) [5].

XAS based methods. XAS is the only method readily available when using the basic XPEEM instrument installed at a synchrotron beamline with a monochromator in place. Among the variety of detection methods to measure X-ray absorption [18], the secondary photoelectrons are collected in XPEEM as a close approximation to the total photoelectron yield measurement. The local XAS spectra are obtained by acquiring image sequences as a function of the photon energy, which can then be

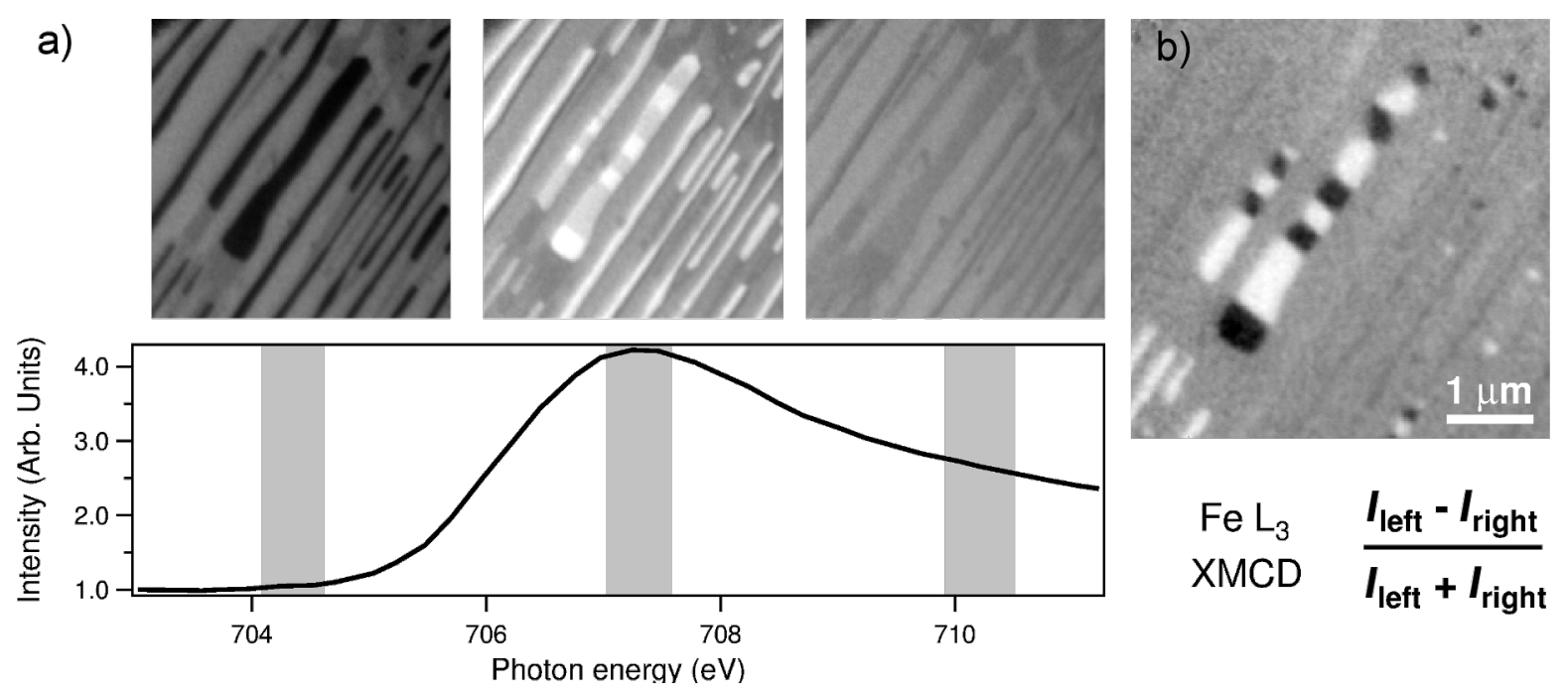

Figure 3: a) Illustration of imaging spectroscopy in XAS mode. Fe nanowires on W(110) appear dark on the left panel at a photon energy of 704.5 eV. At the $\mathrm{Fe}_{3}$ threshold, the wires become much brighter (middle panel). The XAS spectrum below is extracted from the largest nanowire in the center. b) Illustration of XMCD-PEEM imaging. The photon energy is tuned to the $\mathrm{L}_{3}$ maximum. The field of view is $5 \mu \mathrm{m}$. The start voltage is $3 \mathrm{eV}$ in order to collect secondary electrons. Within the image plane, the X-ray direction is perpendicular to the nanowire axis. 
processed in order to extract the intensity variation within any region of interest in the image. Figure 3a illustrates XAS-PEEM imaging spectroscopy on a nanostructured Fe film on W(110). The off-resonant image contrast (leftmost panel) is due to the different secondary photoelectron yield from different surface structures, dominated by the variations in the work function. When the photon energy is tuned to the Fe absorption threshold (middle panel), the elongated Fe nanowires become much brighter, whereas the regions in between barely change intensity. The spectrum seen in the plot in Figure $3 a$ is extracted from an individual nanowire.

Magnetic imaging. X-ray magnetic circular and linear dichroism techniques applied to magnetic surfaces constitute major branches of XPEEM research at synchrotrons. Aside from the photon energy, undulator sources provide also the possibility to manipulate the X-ray polarization. The scattering of circularly polarized X-rays is known to carry a contribution from magnetization, which is greatly enhanced at energies corresponding to certain absorption thresholds [19]. Therefore, XPEEM images can be used to obtain the magnetization distribution on a magnetic surface by a simple polarization analysis [20].

The XMCD-PEEM imaging is illustrated in Figure 3b, in which the magnetization distribution of Fe nanowires on $\mathrm{W}(110)$ is mapped along the beam direction corresponding to the $[1 \overline{1} 0]$ substrate axis. The photon energy is tuned to the $\mathrm{Fe}_{3}$ absorption threshold maximum at about $707.5 \mathrm{eV}$, and the XMCD image is obtained by taking the difference of the two images with opposing circular polarizations. The wires with magnetization along the perpendicular direction, [001] axis, appear gray as they do not produce an XMCD signal. The black and white regions evident in the central wires are the dipolar domains with magnetization parallel and antiparallel to the beam direction, respectively. Note that the strong magnetic contrast in the case of Fe nanowires is visible even in the single XAS image acquired with circular polarization as seen in Figure 3a (middle panel).

Core and valence band spectroscopy. Contrary to XAS-based PEEM, X-ray photoelectron spectroscopy requires a filtering of the kinetic energy of the photoemitted electrons [21]. Therefore, in order to implement XPS in an XPEEM, an energy analyzer has to be installed in the imaging column of the microscope. The most advanced PEEM experiments show a duality between imaging and diffraction operations. The real space image gives a map of the photoelectron intensity, whereas at the backfocal plane the angular distribution of the photoelectrons are imaged. The latter gives access to photoelectron diffraction or angleresolved photoemission from a micrometer-sized region selected by a field-limiting aperture, which we will refer to as $\mu$-ARPES.

Dark-field PEEM is the analogue of the dark-field method in LEEM, such that the same contrast aperture (i.e., diffractionplane aperture) is used to filter out everything except the emission along a given angle. From a practical standpoint, the main difference of the dark-field XPEEM operation is the necessity to change the sample tilt angle in order to get the diffraction feature through the aperture [22,23]. The angular resolution in the dark-field XPEEM is determined by the size of the contrast aperture, which is typically a fraction of an inverse $\AA$. The lateral resolution is comparable to that of the normal XPEEM operation, well below the micrometer scale. Therefore, darkfield XPEEM makes it possible to probe the electronic structure of small features, which cannot be distinguished in the $\mu$-ARPES mode.

\section{The SPELEEM at Elettra}

Although LEEM and PEEM are widespread, only few instruments that combine both methods can be found in synchrotrons. Some prominent ones are situated at ALBA (Spain), BESSY (Germany), Diamond (UK), MAXLAB (Sweden), NSLS (USA), SOLEIL (France) and SPRING-8 (Japan). Among these, the end station of the Nanospectroscopy beamline at Elettra, the 3rd generation storage ring in Trieste, hosts a spectroscopic photoemission and low energy electron microscope (SPELEEM) [24]. This microscope is the commercial evolution (Elmitec GmbH, SPELEEM III) of the first prototype LEEM with a $120^{\circ}$ separator and an energy analyzer, which has pioneered cathode lens spectromicroscopy measurements at synchrotrons during the mid-1990's [25]. The SPELEEM combines LEEM and XPEEM with energy filter in the same setup: LEEM operation uses an $\mathrm{LaB}_{6}$ electron gun and dedicated illumination optics with three condenser lenses, which can deliver a well-collimated e-beam on the sample. In XPEEM operation, instead, the sample is illuminated by the monochromatized X-ray beam produced by the insertion device in the synchrotron ring.

A photograph of the experimental apparatus is shown in Figure 4. Traces indicating the optical path of the incident and scattered beams in LEEM and XPEEM modes are superimposed onto the photograph. Labels indicate the main electronoptical elements and other essential components. The X-ray beam, traced out as a (red) dashed line entering from the right hand side, is incident on the sample at a $16^{\circ}$ grazing angle from the surface plane. The backscattered/emitted electrons are accelerated to $18 \mathrm{keV}$ towards the electromagnetic objective lens. Next element along the optical path is the beam separator, which deflects the e-beam towards the imaging column in 
which a magnified image of the sample is produced. Depending on the lens excitations, the diffraction pattern at the objective lens backfocal plane can be imaged. At the entrance of the energy analyzer, the electrons are decelerated from $18 \mathrm{keV}$ to $908 \mathrm{eV}$, the pass energy of the filter. Upon exit from the analyzer, the e-beam is re-accelerated to $18 \mathrm{keV}$. The final image is projected onto the detector, a chevron multichannel plate followed by a phosphorous screen. The light produced in the phosphorous screen is collected by a CCD camera (PCO Sensicam QE) equipped with an external fan for vibrationless cooling. A more detailed explanation of the SPELEEM optics can be found in reference [25].

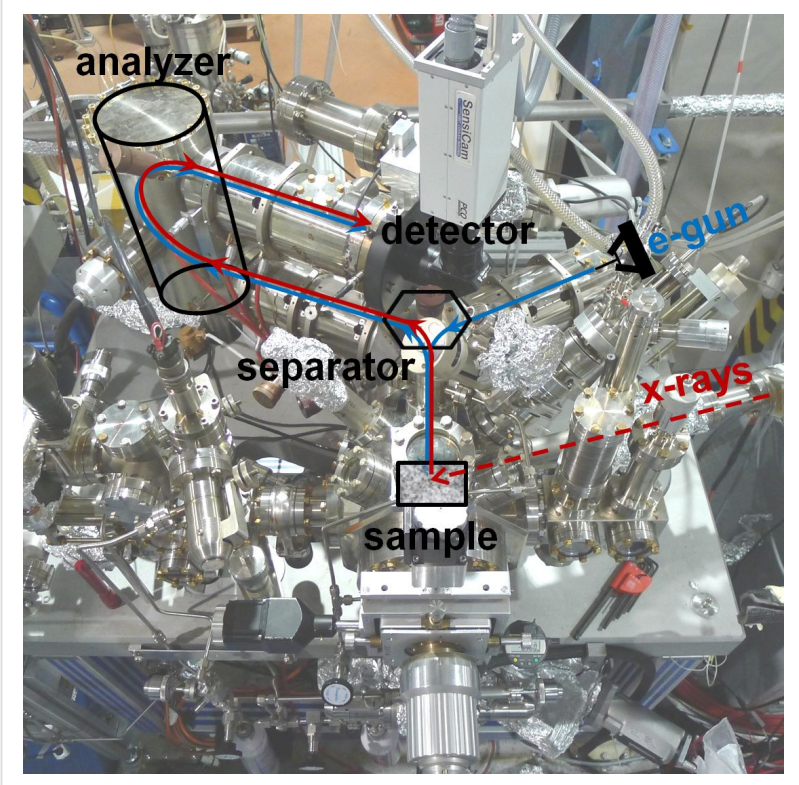

Figure 4: The SPELEEM instrument at the Nanospectroscopy beamline, Elettra Sincrotrone, Trieste. The sketch of the basic setup is superimposed onto the photograph. X-rays arrive from the right at $16^{\circ}$ grazing angle to the sample surface.

Electron source. The SPELEEM is equipped with an $\mathrm{LaB}_{6}$ thermionic emission cathode (Kimball Physics, model ES-423E, style 06-60). The emitter is a single crystal cut to a cone angle of $60^{\circ}$ exposing a $\langle 100\rangle$ microfacet of $6 \mu \mathrm{m}$ diameter as tip, which offers high electron flux at a relatively low temperature of the emitter. In the SPELEEM instrument, the electron beam

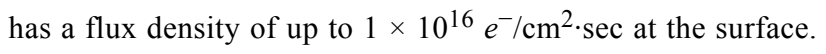
The illumination optics focus the beam on the sample to a slightly elliptical shape with diameter of about $90 \mu \mathrm{m}$.

The energy spread of the $\mathrm{LaB}_{6}$ source is set by its operation temperature, reaching $1900 \mathrm{~K}$ at a current of $2.12 \mathrm{~A}$. Figure $5 \mathrm{a}$ shows the energy distribution of the electron source at the SPELEEM instrument for an operation current of $1.75 \mathrm{~A}$. In order the determine the emitter characteristics, we fitted the experimental data modeling the energy dependence with a simple function taking into account instrumental and thermal broadening [26]. The long tail in the energy dependence of the intensity seen in Figure 5a reflects the $\mathrm{LaB}_{6}$ temperature. By fitting the curve with a Fermi function, we determine a tip temperature of about $1750 \mathrm{~K}$. The sharp rise on the left hand side represents the effect of the instrumental broadening. The broadening obtained from this leading edge is about $65 \mathrm{meV}$, providing a best estimate for the energy resolution of our electron energy analyzer in LEEM operation. Note that this figure is not limited by the size of the contrast aperture, as the angular spread in LEEM is smaller than the aperture size.
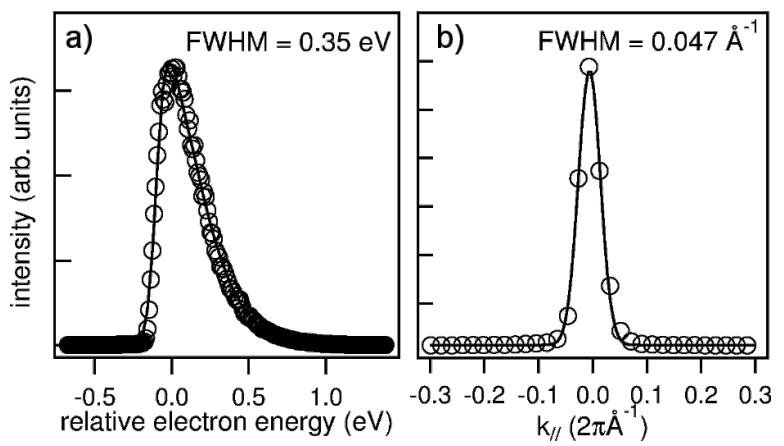

Figure 5: a) The energy distribution of the electron beam emitted from the $\mathrm{LaB}_{6}$ source acquired by keeping the sample below the MEM transition using a negative start voltage bias. The intensity-vs-energy curve is obtained in dispersive plane operation, in which the exit plane of the energy analyzer is projected onto the detector. b) The (00) LEED spot profile from $\mathrm{W}(110)$.

The transfer width of the system was measured from the profile of the (00) specular diffraction spot originating from a virtually step-free region of a W(110) crystal, as shown in Figure 5b. Under normal operating conditions, when using a $2 \mu \mathrm{m}$ illumination aperture and $0.05 \mu \mathrm{A}$ total emission from the e-gun, the full width half maximum of the Gaussian spot profile was found to be $0.047 \AA^{-1}$. This corresponds to a transfer width of more than $130 \AA$ in real space.

X-ray source. Two Apple-II type undulators provide an intense $\mathrm{X}$-ray beam with linear horizontal, linear vertical, or circular polarization from below $10 \mathrm{eV}$ up to $1000 \mathrm{eV}$ [24,27,28]. The monochromator makes use of three gratings to cover the entire photon energy range. A spherical grating is used at the low energies below $50 \mathrm{eV}$, whereas two variable line spacing gratings of 200 lines $/ \mathrm{mm}$ and 400 lines $/ \mathrm{mm}$ cover the ranges of 50-250 eV and 250-1000 eV, respectively. The footprint of the $\mathrm{X}$-ray beam is about $20 \times 5 \mu \mathrm{m}^{2}(H \times V)$, horizontally elongated because of the grazing incidence. A larger area (up to $30 \mu \mathrm{m}$ ) can be illuminated by slightly defocusing the photon beam and by moving it along vertically during image acquisi- 
tion, although this method usually causes striations in the illumination. The maximum flux is obtained at about $150 \mathrm{eV}$ photon energy and is about $1.8 \times 10^{13}$ photons/s with the exit slit of the monochromator set to $10 \mu \mathrm{m}$ and with $200 \mathrm{~mA}$ synchrotron ring current [29].

Energy resolution in XPEEM. The photon source and the SPELEEM operation mode together determine the value of the energy resolution. The resolving power of the VLS400 grating of the beamline monochromator, $E / \Delta E$, is about 3000 for the photon energy range from 700 to $1000 \mathrm{eV}$. This figure nearly doubles at lower photon energies [30]. Therefore, in the majority of practical cases, the microscope energy analyzer is the limiting factor in terms of energy resolution.

In energy-filtered imaging and diffraction, where images are collected at a well-defined photoelectron kinetic energy, the energy resolution is mainly determined by the size of the analyzer exit slit. Two slits of different width can be used to set the bandpass of the energy filter, corresponding to energy windows of $0.33 \mathrm{eV}$ and $0.79 \mathrm{eV}$, respectively. Note that the best energy resolution from the instrument can be obtained in the $\mu$-spectroscopy operation, in which the dispersive plane of the analyzer is imaged onto the detector. In this way, the resolution is mainly determined by the radius of the analyzer hemisphere $\left(r_{0}=10 \mathrm{~cm}\right)$, the pass energy $(E=908 \mathrm{eV})$, and the angular spread before the analyzer ( $\alpha=5 \mathrm{mrad}$ full width) given by the size of the contrast aperture $(d=20 \mu \mathrm{m}$, smallest aperture), which also acts as the entrance slit. The energy spread $\Delta E$ (full width at half maximum) is parameterized as [31]:

$$
\Delta E=E\left(0.43 \frac{d}{r_{0}}+\alpha^{2}\right)
$$

Inserting the values listed above, the best resolution is estimated to be $101 \mathrm{meV}$. The experimental energy resolution of the SPELEEM was measured from the $\mathrm{W} 4 \mathrm{f}$ core level of the clean W(110) surface. The dispersive plane spectrum and the corresponding Doniach-Šunjić fit are displayed in Figure 6 [32]. The resulting full-width half-maximum of the Gaussian broadening is found to be less than $110 \mathrm{meV}$ for the optimal conditions (smallest contrast aperture, low photon flux, small field-limiting aperture), which is in excellent agreement with the above estimation. For the usual operating conditions with less stringent parameters, the energy resolution was found to be about $150 \mathrm{meV}$.

Lateral resolution. The SPELEEM spatial resolution is mainly determined by the spherical and chromatic aberrations of the objective lens. LEEM performs better compared to XPEEM.

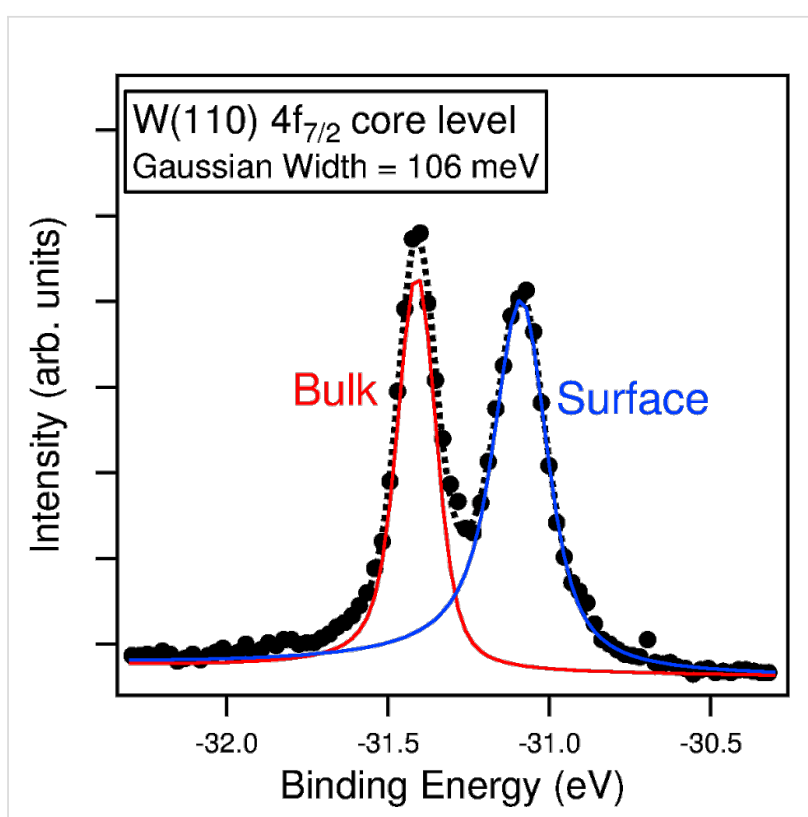

Figure 6: Tungsten $4 f_{7 / 2}$ core level spectrum from a clean $W(110)$ surface acquired in dispersive plane mode. The photon energy is $90 \mathrm{eV}$. The acquisition time is $80 \mathrm{~s}$. The Lorentzian broadening for the bulk peak was fixed at $60 \mathrm{meV}$, with an asymmetry parameter of 0.035 . The contrast aperture, which acts as the analyzer entrance slit, is $20 \mu \mathrm{m}$.

Low energy electron diffraction beams are generally much sharper than the broad photoelectron emission angles. As a result, the angular spread (thus spherical aberrations) in LEEM is considerably reduced compared to XPEEM. The same is true also for the energy spread and the chromatic aberrations, which are again reduced in the case of LEEM. The lateral resolution in LEEM mode for the SPELEEM at Nanospectroscopy is demonstrated in Figure 7. The plot shows the variation of the LEEM intensity across a profile through a ML thick Ni island on $\mathrm{W}(110)$, along with a sigmoid fit. The width of the sigmoid represents the instrumental broadening, corresponding to the distance identified by using the $16-84 \%$ intensity variation criterion often used to characterize the lateral resolution. By averaging over several profiles across the image, we obtained a value of about $9 \mathrm{~nm}$. The same value is obtained when measuring the width of steps (providing phase instead of amplitude contrast) on clean W(110). Note that this value is better by more than $20 \%$ compared to the performance prior to the installation of the new electron source. This improvement is based on the smaller energy spread and the reduced transfer width, which are reported in Figure 5. The lateral resolution may possibly be further reduced by using a field-emission source with superior characteristics.

The spatial resolution in XPEEM is about $30 \mathrm{~nm}$ [24]. For comparison, consider that the wide angular spread observed when 


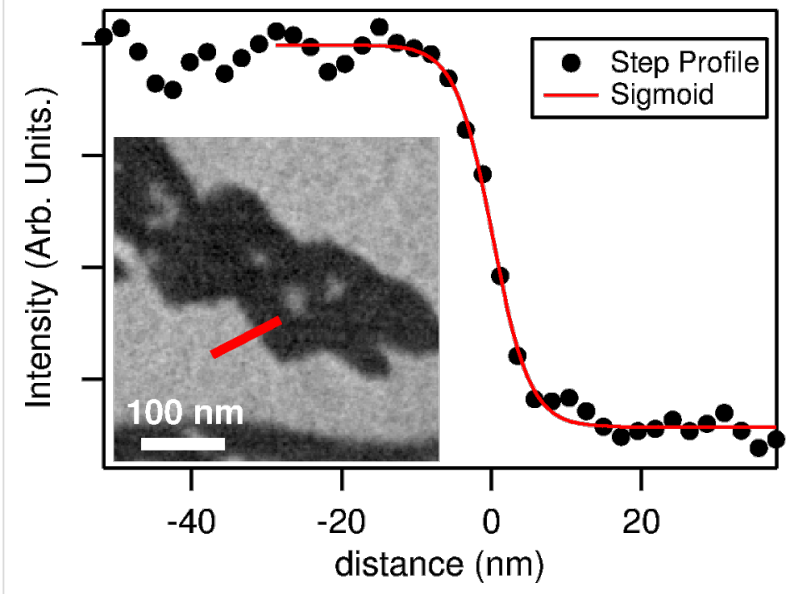

Figure 7: Lateral resolution in LEEM. The inset shows a Ni monolayer island (dark) on $\mathrm{W}(110)$. The profile in the plot is marked on the image. The full width of the sigmoid function is $8.2 \mathrm{~nm}$. Averaging over several profiles, the value is found to be $8.6 \pm 1.2 \mathrm{~nm}$.

imaging the inelastically scattered energy-loss electrons in LEEM gives a spatial resolution of about $18 \mathrm{~nm}$, similar to XPEEM [33]. It is important to note that the lateral resolution in XPEEM suffers from space-charge effects due to the highly brilliant synchrotron pulses [29]. The electron-electron interaction within the photoelectron pulse produced by the X-ray pulse (which has a low duty cycle of about $1 / 50$ ) results in the degradation of the image quality as well as increasing the energy spread. As a result, even the aberration-corrected instruments are limited to a moderate lateral resolution in XPEEM [34]. Similar effects were not observed in LEEM with its monochromatic $\mathrm{LaB}_{6}$ cathode providing a much lower current density than the peak photoelectron current in XPEEM.

\section{SPELEEM studies of graphene epilayers}

LEEM has found ample use in graphene research with its high structural sensitivity and video acquisition rate allowing for dynamic measurements of film growth. In such experiments, graphene is typically obtained by the chemical vapor deposition (CVD) technique. CVD utilizes transition metal catalysts as a means to promote the dissociative adsorption of gases such as ethylene or methane, which can readily deliver the carbon atoms required for island nucleation and growth. LEEM is widely employed to image the growth process; the accessible parameter space is explored by varying the gas pressure and/or sample temperature in a manner compatible with the operation limits of the instrument. In this regard, LEEM permits operation at elevated sample temperatures (approximately up to $1000^{\circ} \mathrm{C}$ ) by using the experimental chamber as a gas flow reactor up to pressures approaching $1 \times 10^{-5} \mathrm{mbar}$, still ensuring a lateral resolution nearing $10 \mathrm{~nm}$. The growth of graphene on a variety of transition metal substrates provide catching examples of the potential of the LEEM method [3537]. For instance, by measuring subtle variations in the low energy electron reflectivity of the substrate upon ethylene uptake, it has been shown that LEEM can quantitatively monitor the time evolution of the $\mathrm{C}$ adatom lattice gas that anticipates island nucleation. Tiny variations of the carbon coverage were detected in these experiments, achieving sensitivities below $0.1 \%$ of a ML. These experiments provided a formidable means to access to the thermodynamics governing carbon segregation, graphene nucleation and film growth [3638]. In other cases, LEEM imaging was used to monitor the intercalation of adspecies below graphene [39-41]. As a further advantage, LEEM allows for thickness determination in multilayer systems, through the exploitation of quantum size contrast. Nevertheless, the understanding of the low energy electron reflectivity is not always straightforward, since it is extremely sensitive to the substrate-film interaction, as has been recently demonstrated by an ab initio study that clarified the interpretation of LEEM- $I(V)$ curves [42].

An important aspect is the integration of LEEM with low energy electron diffraction (LEED). Diffraction experiments permit a full characterization of the crystal structure and quality of graphene. For instance, $\mu$-LEED methods have been devised to quantify the short-range roughness of multi-thickness $\mathrm{SiO}_{2}-$ supported and suspended exfoliated graphene films [43]. To date, the most frequent application of LEEM/LEED has been the study of rotational domains and complex moiré patterns in a wide variety of graphene epilayers. A notable example is that of graphene on $\operatorname{Re}(0001)$, in which a moiré cell made out of $(10 \times 10)$ graphene unit cells over $(9 \times 9)$ Re unit cells was determined in a $\mu$-LEED experiment performed in a SPELEEM microscope; the atomic positions in the unit cell were subsequently obtained by means of ab initio calculations, which could prove the very large corrugation of graphene in this system and establish a correlation between $\mathrm{C} 1 \mathrm{~s}$ binding energy and $\mathrm{C}$-substrate separation [44]. On the same system, $\mu$-probe diffraction analyses were carried out in combination with darkfield imaging, investigating the carburisation of the $\operatorname{Re}(0001)$ substrate as a function of temperature [45]. The literature shows a plethora of other experiments exploiting the LEED capabilities of LEEM with notable examples of graphene on single and polycrystalline copper [46-48], nickel [49] and on non-threefold crystalline substrates such as $\operatorname{Ir}(100)[50,51]$ and $\mathrm{Fe}(110)$ [52].

There is also a growing literature on XPEEM applications in graphene research. In particular, $\mu$-ARPES available in the SPELEEM has been successfully applied to exfoliated [53,54] as well as epitaxial graphene grown on metal $[48,55,56]$ and 
$\mathrm{SiC}$ substrates [57]. In many studies $\mu$-ARPES was employed to access the $\pi$-band of graphene, to quantify the doping in graphene and to verify the strength of the film-substrate interaction. At length scales below the resolution of the $\mu$-probe approach, the recently introduced dark-field XPEEM method is ideally suited to compare the density of states (DOS) of different, adjacent types of graphene exhibiting distinct electronic structure properties. Laterally resolved XAS was also utilized in an isolated study on exfoliated graphene, in which selected features in the $\mathrm{K}$-edge spectrum of $\mathrm{C}$ were studied as a function of the thickness of graphene. A splitting of the $\pi^{*}$ resonance was observed in multilayers and ascribed to specific interlayer states [58].

The intercalation of Au below graphene on $\operatorname{Ir}(100)$. Recently investigated by using a wide range of microscopy methods and theory, the graphene/Ir(100) system exhibits unique morphological and electronic properties, which originate from the different film and substrate symmetries [50]. At temperatures above $800{ }^{\circ} \mathrm{C}$, micrometer-sized single layer graphene crystals can be obtained upon exposure to ethylene, oriented at $3^{\circ}$ with respect to the main substrate direction. By cooling the sample from growth to room temperature, a phase transformation occurs in the graphene film, which develops neighboring phases characterized by flat and buckled morphology. Adjacent stripedshaped domains of different carbon surface density alternate on the film at microscopic length-scales, relieving the strain accumulated upon cooling to room temperature. Most interestingly, the buckled graphene phase is characterized by large and extremely regular one-dimensional ripples showing a periodicity of $2.1 \mathrm{~nm}$. Dark-field PEEM experiments have demonstrated that the buckled graphene phase shows a negligible DOS at the $K$ point of the $\pi$-band. These results point to the disruption of the Dirac cones and the formation of a metal-like DOS. Surprisingly, the hybridization of the $\pi$-band with Ir states is due to the chemisorption of just $11 \%$ of the $\mathrm{C}$ atoms in the unit cell of the buckled phase [50].

In order to modify the graphene-substrate interaction in this system, we have intercalated $\mathrm{Au}$ at elevated sample temperature, taking advantage of the fast diffusion of Au adatoms under such conditions. Au is expected to exert a weaker interaction than Ir, hindering the formation of the chemisorption bonds such as those observed in the buckled graphene phase on $\operatorname{Ir}(100)$. In our work, the related variations in $\mathrm{C}-$-substrate bonding and the electronic structure of graphene were quantified in $\mu$-XPS and $\mu$-ARPES experiments, taking the pristine graphene/Ir(100) system as reference. Experimentally, Au was deposited by using an e-beam evaporator (Elmitec $\mathrm{GmbH}$ ) at a flux of $0.059 \mathrm{eML}_{\operatorname{Ir}(100)}$ (equivalent monolayer of $\operatorname{Ir}(100)$ ) per minute at a sample temperature of about $600{ }^{\circ} \mathrm{C}$.
The evolution of the interface upon increasing Au dose was monitored in situ employing LEEM. Figure 8a illustrates the initial state of the surface, with a graphene island (bright) located in the lower half of the image. The same image (upper half) shows the bare Ir surface, rendered in medium gray; a few thin curved lines can be spotted here, which identify morphological features of the surface such as steps and step bunches. The first stages of the $\mathrm{Au}$ growth are shown in Figure 8b, the Au-covered areas appearing darker than the Ir substrate at the chosen electron energy $(12 \mathrm{eV})$. As can be seen, Au has already decorated the steps and has formed a step-flow growth front, its local thickness being just one layer. Note that $\mathrm{Au}$ is not found on or below graphene, but adsorbs exclusively on the iridium substrate. Only when the bare Ir surface has been fully covered, the intercalation process starts. At this stage, the Au growth front propagates quickly under the film, as shown in Figure 8c and Figure 8d, until a full Au monolayer is formed below graphene.

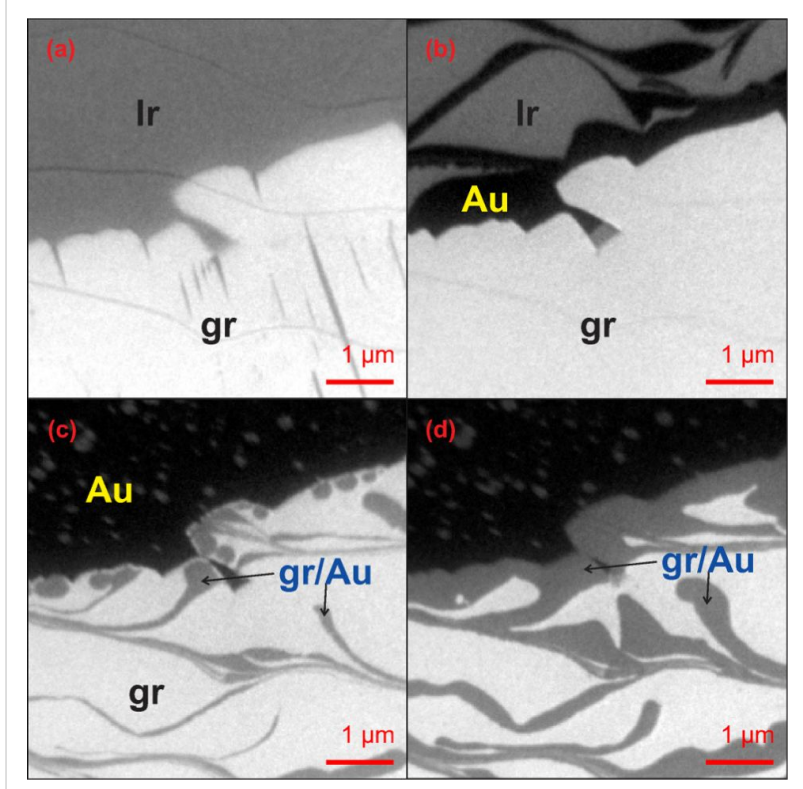

Figure 8: LEEM images at a start voltage of $12 \mathrm{eV}$ illustrating the evolution of the graphene/ $/ \mathrm{r}(100)$ interface upon deposition of $\mathrm{Au}$. A large graphene crystal (gr) is visible in all images (lower half), brighter than the Ir surrounding it (upper half). (a) Initial configuration of the sample at $T=520^{\circ} \mathrm{C}$. (b) The same area after a dose of $0.25 \mathrm{ML}$ Au at sample temperature of $600{ }^{\circ} \mathrm{C}$. Au (dark areas) has decorated steps and step bunches. (c) The same area after a dose of $0.85 \mathrm{ML}$ of Au. At this stage, Au has entirely covered the initially bare Ir surface and the intercalation under graphene has just started (darker areas). Note also that small graphene islands have nucleated on the Au/lr surface.

(d) The same area after a dose of $0.9 \mathrm{ML}$ of $\mathrm{Au}$.

There are two interesting findings that need to be highlighted. First, it appears that Au can intercalate below graphene only after accumulation of a full $\mathrm{Au} \mathrm{ML}$ at the island edges, and before the nucleation of second or multilayer $\mathrm{Au}$ islands. 
Second, small graphene islands nucleate at the Au-covered surface far from the large graphene crystal, as was verified by $\mu$-LEED analysis. The former observation gives the order of energetics for Au adsorption on Ir versus its intercalation under graphene. The intercalation is facilitated by the lifting of the chemisorbed graphene edges upon Au adsorption at the edges. To explain the appearance of small graphene islands on $\mathrm{Au}$, we must consider that $0.1 \mathrm{ML}$ carbon is chemisorbed to the $\mathrm{Ir}$ surface, forming a carbidic $\mathrm{c}(2 \times 2)$ structure [51]. In such a structure, $\mathrm{C}$ is strongly bound to the substrate, with a binding energy of almost $8 \mathrm{eV}$ at a coverage of $0.5 \mathrm{eML}_{\operatorname{Ir}(100)}$ [59]. We note, however, that $\mathrm{Au}$ binds strongly to other noble metal substrates. For instance, on $\mathrm{Rh}(110)$ the binding energy of $\mathrm{Au}$ is $3.5 \mathrm{eV}$ at ML-coverage [60]. Due to the strong interaction with $\mathrm{Ir}$, the adsorption of $\mathrm{Au}$ weakens the $\mathrm{C}-\mathrm{Ir}$ bonds, and the high density of $\mathrm{C}$ adatom gas on the Au layer readily condense to form graphene islands.

Upon subsequent cooling to room temperature, the morphology and structure of graphene remain unchanged. In fact, we could not detect any evidence of phase transformation or formation of stripe-shaped domains resembling those of observed on $\operatorname{Ir}(100)$. Instead, LEEM imaging at high lateral resolution evidenced the formation of wrinkles in the graphene film, a process which helps relieving the thermal strain, because of the different thermal contraction of film and substrate. Similar features have been previously observed for graphene on $\operatorname{Pt}(111)$ and $\operatorname{Ir}(111)$ surfaces $[61,62]$. Importantly, no coincidence structures are observed in the LEED pattern, which exhibits only the first order graphene spots plus an extremely week moiré structure, identical to that observed on the flat phase of graphene on $\operatorname{Ir}(100)$. This finding further confirms that, after Au intercalation, graphene is entirely physisorbed and no chemisorption bonds are established between $\mathrm{C}$ and the substrate.

The C 1s $\mu$-XPS spectrum of the graphene/ $\operatorname{Ir}(100)$ system exhibits two components. The dominant one, at about $283.95 \mathrm{eV}$ binding energy, has been previously ascribed to physisorbed C [50], consistent with the binding energy values observed for weakly-interacting graphene on a variety of fcc(111) and hcp(0001) substrates [44,63]; the second peak appears at higher binding energy (about $284.9 \mathrm{eV}$ ) and is due to the small fraction of chemisorbed carbon atoms in the buckled phase. Conversely, the C 1s spectrum measured on the graphene/Au system shows only the physisorbed component, proving that no chemisorption to the substrate has been established upon cooling to room temperature.

Clearly, the Au layer induces important variations in the graphene-substrate interaction, which in turn affect the charge transfer processes occurring between substrate and film and, consequently, the doping. Figure 9 shows $\mu$-ARPES patterns (a, top) and momentum distribution curves (b, bottom) along the high symmetry directions for the graphene/Au system (on the left-hand side of the Figure) and pristine graphene on $\operatorname{Ir}(100)$ (on the right-hand side), respectively. Visual inspection of the full $\mu$-ARPES pattern at $E_{\mathrm{F}}$ shown in (a) instantly reveals that $\mathrm{Au}$ intercalation is manifested by a change in the doping: The circular features identifying the Dirac cones for the graphene/ $\mathrm{Au}$ system appear narrower than those recorded on pristine graphene, suggesting that the Dirac energy is now closer to the Fermi level. By accurately fitting of momentum distribution curves we could determine a positive doping of just $0.09 \pm 0.06 \mathrm{eV}$ for graphene/ $\mathrm{Au} / \operatorname{Ir}(100)$, which has to be compared with the value of $0.42 \pm 0.03 \mathrm{eV}$ obtained on graphene/Ir(100) [50]. Our results are in fair agreement with $\mu$-ARPES data for the graphene/Au/Ni(111) system [64]. On the $\mathrm{Ni}$ substrate, the Au intercalation leads to a non-rigid shift of the bands of graphene towards lower binding energies, the $\pi$-band moving by approximately $2 \mathrm{eV}$; the Dirac energy $E_{\mathrm{D}}$ is found at just $25 \mathrm{meV}$ above the Fermi level, so that quasi freestanding conditions for the film are claimed. Referring to an analytical model $[65,66]$, the Fermi level shift in graphene translates to the graphene-Au distance beng in the range of 3.4-3.6 $\AA$, slightly larger than the calculated graphene-Ir distance $[50]$.

\section{(a) ARPES}

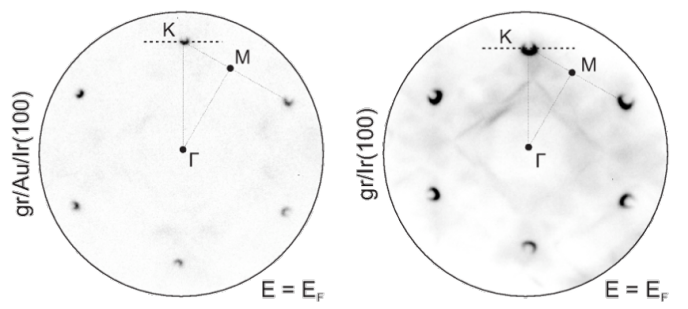

(b) Momentum Distribution Curves

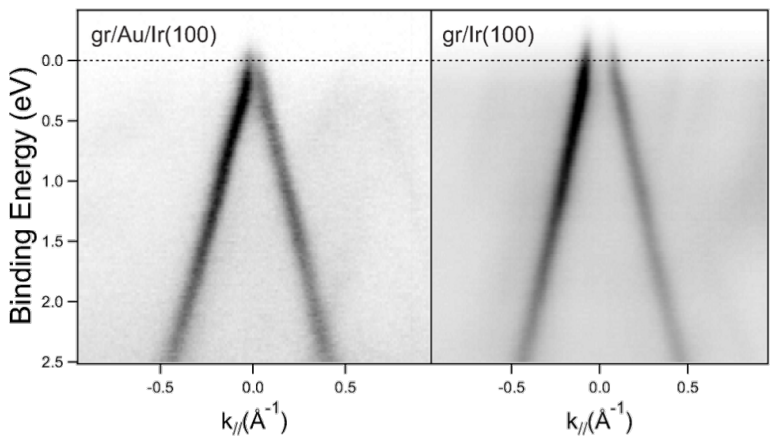

Figure 9: Graphene on Au/lr(100) (left column) and Ir(100) (right column). (a) $\mu$-ARPES near $E_{F}$; the high symmetry points in the first Brillouin zone (FBZ) are indicated. Photon energy is $40 \mathrm{eV}$. The probed area has a diameter of $2 \mu \mathrm{m}$. (b) Momentum distribution curves along the normal to the $\Gamma-\mathrm{K}$ direction, as indicated by the dashed line in (a). 


\section{Self-organized nanomagnets}

Self-organization may be ascribed the general meaning "spontaneous appearance of a particular form". Even though the definition may be stretched about to describe nearly all observed shapes in nature, static and dynamic, we assign the term to the formation of regular structures. SPELEEM methods perfectly lend themselves to studies of self-organization phenomena, particularly in the field of nanomagnetism. In a nutshell, LEEM is used to monitor the growth process in real time at high temperatures; spectromicroscopy with XPEEM provides the chemical map of the resulting heterogeneous surface; and finally XMCD-PEEM reveals the magnetization distribution of this nanostructured surface.

Stress-induced adsorbate stripes have been recently observed on crystalline surfaces at high temperatures. The mechanism is based on the competition between the cost of a boundary and the gain due to long range elastic interactions between boundaries [67]. The temperature determines the relative strength of the long- and short-ranged energy terms [68].

In the case of monolayer Pd stripes on W(110) forming at about $1100 \mathrm{~K}$ [68], it was recently shown that the addition of oxygen modifies the pattern anisotropy while preserving the periodic structure [69]. Moreover, the $\mathrm{Pd}-\mathrm{O}$ bispecies layer is stable upon lowering the temperature from above $1000 \mathrm{~K}$ down to room temperature. The different adlayer patterns that can be obtained by varying the amount of oxygen on the surface are depicted in Figure 10a. The changes in the pattern anisotropy are driven by the magnitude and sign of stress variations on the surface, which are both dependent on the presence and amount of oxygen [69].

The stability of the $\mathrm{Pd}-\mathrm{O}$ stripes on $\mathrm{W}(110)$ at lower temperatures allows for a further growth of magnetic wires following the self-organized template. $\mathrm{Fe} / \mathrm{Pd}-\mathrm{O}$ stripes on $\mathrm{W}(110)$ have been demonstrated very recently [70]. XPEEM imaging at the $\mathrm{Fe}_{3}$ absorption threshold has confirmed the expected $\mathrm{Fe}$ distribution as seen in Figure 10b (left panel). In the resulting picture, Fe preferentially wets the Pd-covered parts of the striped template. Furthermore, at about $200{ }^{\circ} \mathrm{C} \mathrm{Fe}$ and $\mathrm{Pd}$ rearrange to make a surface alloy with a Pd-rich surface layer. The magnetization of the FePd stripes was found to be along the [1 $\overline{1} 0]$ direction perpendicular to the stripe axis, as shown in the XMCD map in Figure 10b (right panel). This is a surprising confirmation of the magnetocrystalline anisotropy strength dominating the shape anisotropy.

Iron oxides find wide application in several fields of research, among others magnetism and heterogeneous catalysis. In both cases, the heteroepitaxial growth of nanostructured $\mathrm{FeO}_{x}$ offers

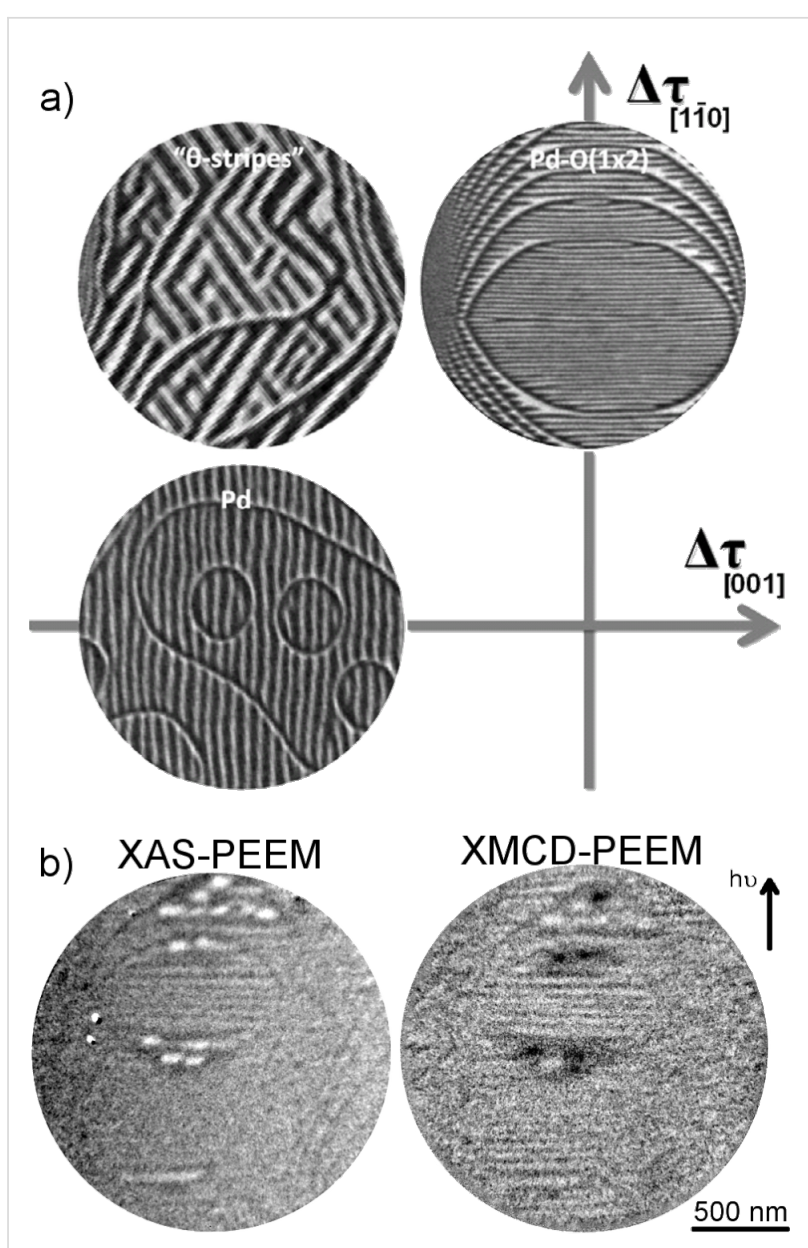

Figure 10: a) LEEM images ( $2 \mu \mathrm{m}$ diameter) of monolayer $\mathrm{Pd}$ stripes on $\mathrm{W}(110)$. The lower left panel shows Pd on a clean substrate, whereas the other two panels display the progressive change in pattern anisotropy upon addition of $0.1 \mathrm{ML}$ and $0.33 \mathrm{ML}$ oxygen. (Reprinted from [69]. Copyright 2011 IOP Publishing.) b) Fe grown on $\mathrm{Pd}-\mathrm{O}$ stripes at $225^{\circ} \mathrm{C}$. Left panel is the XAS-PEEM image at the $\mathrm{Fe}$ $\mathrm{L}_{3}$ edge showing the Fe distribution. On the right, the Fe XMCD image indicates that the wires are uniformly magnetized perpendicular to the long axis. (Reprinted from [70]. Copyright 2013 Elsevier.)

a versatile means to tune the material properties. Recently, SPELEEM techniques were applied to characterize the reactive growth of $\mathrm{FeO}_{x}$ on $\mathrm{Ru}(0001)$ [71]. Fe growth in an oxygen ambient $\left(5 \times 10^{-7}\right.$ mbar $)$ at $900 \mathrm{~K}$ resulted in the formation of perfectly triangular micrometer-sized $\mathrm{Fe}_{3} \mathrm{O}_{4}$ islands on a $\mathrm{FeO}$ wetting layer. The combination of spatially-resolved XPS and XAS spectra, along with $\mu$-LEED patterns, allowed the unequivocal identification of the specific iron-oxide phases.

From the screening of substrate core-level photoelectrons, the thickness of the micrometer-sized magnetite islands was found to be about $1 \mathrm{~nm}$, which corresponds to two unit cells [72]. XMCD-PEEM measurements on these ultrathin islands (seen in Figure 11a) show that magnetite preserves its ferrimagnetic properties at $1 \mathrm{~nm}$ thickness up to $520 \mathrm{~K}$ (above which the 

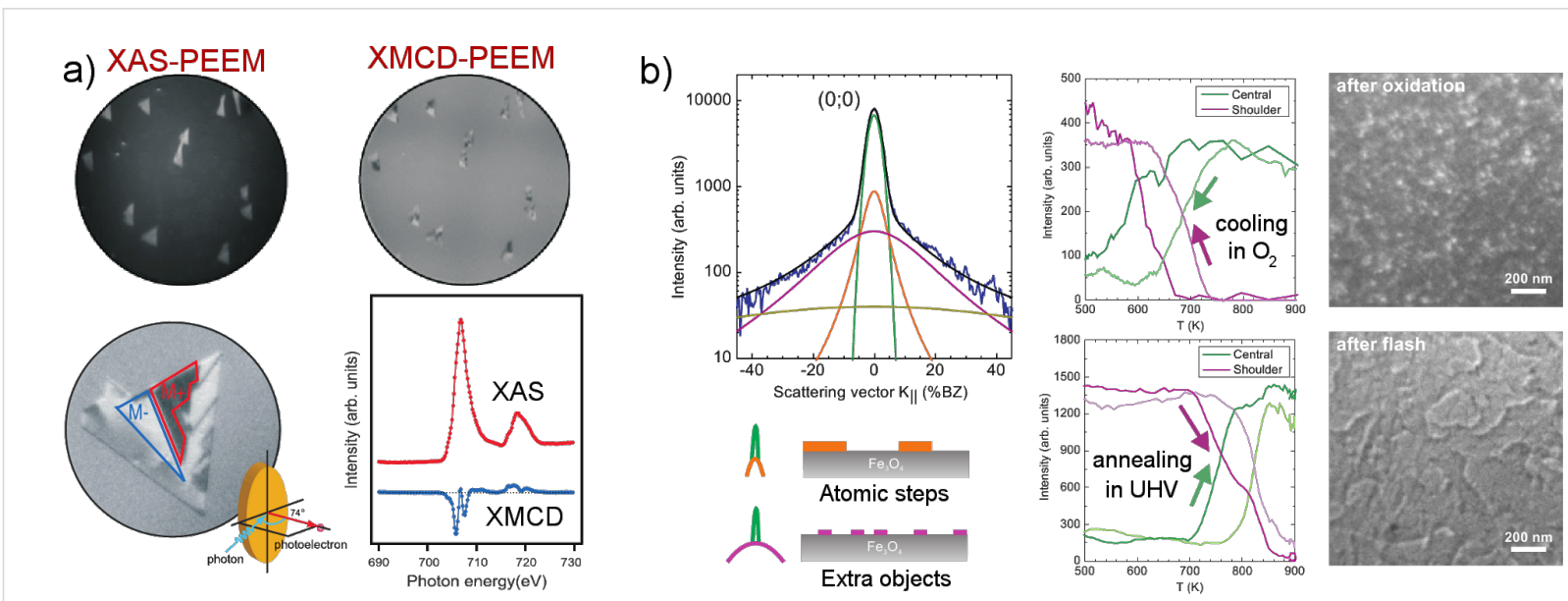

Figure 11: a) Magnetite islands and the $\mathrm{FeO}$ wetting layer on $\mathrm{Ru}(0001)$. Top panels show the island and magnetization distribution within a region of $30 \mu \mathrm{m}$ diameter, illuminated homogeneously by vertically scanning the photon beam during acquisition. Bottom panels show the details of the magnetic domains (left, field of view $4 \mu \mathrm{m}$ ) and the $\mathrm{Fe} \mathrm{L}_{3}$ XMCD spectrum extracted from a single domain (right). (Reprinted with permission from [72]. Copyright 2012 American Physical Society.) b) Morphology of $\mathrm{Fe}_{3} \mathrm{O}_{4} / \mathrm{Pt}(111)$ from LEEM and $\mu$-LEED spot profile analysis. The large tail in the (00) spot profile (seen on the left) is identified with the formation of oxygen-related agglomorates as sketched below. Middle panels show the effect of cooling/annealing on the spot profile. LEEM images on the right (at an energy of $24 \mathrm{eV}$ ) present the surface before and after the annealing cycle. (Reprinted with permission from [73]. Copyright 2012 American Physical Society.)

morphology changes irreversibly). This observation corresponds to the thinnest magnetite crystal that shows magnetism.

Beyond the self-organized crystal shapes at the micrometer scale, epitaxial iron-oxide films provide a variety of complex surface reconstructions at the atomic scale as usual for oxide surfaces [74]. $\mathrm{Fe}_{3} \mathrm{O}_{4}$ films on $\mathrm{Pt}(111)$ are known to give a $(2 \times 2)$ reconstruction with an additional moiré superstructure. Nevertheless, the details of the $\mathrm{Fe}_{3} \mathrm{O}_{4}$ surface structure is still under study. The recent work by using an aberration-corrected XPEEM-LEEM setup, SMART (BESSY II, Helmholtz Zentrum, Berlin), showed distinct differences between LEED $I(V)$ curves obtained from surfaces with differing preparation pathways [73]. The role of surface preparation was revealed in the LEED spot-profile analysis of the $\mathrm{Fe}_{3} \mathrm{O}_{4}(111)$ surface as summarized in Figure 11b, which showed the formation of oxygen-related defects. In particular, the different contributions to the LEED spot-profile were observed in real-time as a function of temperature, which resulted in a model of oxygeninduced extended surface defects [73].

Aside structure and magnetism, transformations between different iron-oxide phases were studied by using the SPELEEM methods. In the above example of $\mathrm{FeO}_{x}$ growth on $\mathrm{Ru}(0001)$, further oxidation by using $\mathrm{NO}_{2}$ as atomic oxygen source resulted in the transformation of the $\mathrm{FeO}$ wetting layer to hematite $\left(\alpha-\mathrm{Fe}_{2} \mathrm{O}_{3}\right)$ and the triangular $\mathrm{Fe}_{3} \mathrm{O}_{4}$ islands to maghemite $\left(\gamma-\mathrm{Fe}_{2} \mathrm{O}_{3}\right)$ [71]. In an independent study, the realtime observation of thermal reduction with LEEM and LEED was crucial in understanding the reversible changes in thin magnetite and hematite films grown on several substrates [75]. In particular, annealing in UHV led to substrate-dependent transformations of the iron oxide thin film: from hematite to magnetite on a $\mathrm{Pt}(111)$ substrate and vice-versa on $\operatorname{Ag}(111)$. The conversion has been explained as a competition between the dilution of Fe cations in the substrate, predominant only in the former case, and the desorption of oxygen.

\section{Summary}

We have given a review of SPELEEM methods along with recent applications in the fields of graphene and nanomagnetism. The extensive introduction to LEEM and XPEEM techniques illustrates the basic operation principles, with the intention to serve as a guideline for those unfamiliar with the field. A working example of a SPELEEM instrument is the one available at the Nanospectroscopy beamline at Elettra. Details on the instrumental aspects of the Nanospectroscopy microscope have been given both as an update on the performance of this particular setup, and also as a reference for the typical SPELEEM properties regarding parameters such as energy resolution, lateral resolution, electron beam characteristics and the transfer function of the instrument. After the instrumental part, recent scientific activity in graphene research by using LEEM-PEEM methods have been reviewed. Then, the original example of $\mathrm{Au}$ intercalation at the graphene- $\operatorname{Ir}(100)$ interface has been presented, showing the effective role of $\mathrm{Au}$ in breaking the $\mathrm{C}-\mathrm{Ir}$ chemisorption bonds and in restoring the neutral Dirac point nearly at the Fermi level. The review has been concluded with examples on self-organized nanomagnetism studies taking advantage of the possibility to perform magnetic imaging with 
an XPEEM, based on the X-ray magnetic circular dichroism contrast.

\section{References}

1. Septier, A. Geometrical Electron Optics. In Electron Microscopy in Material Science; Valdrè, U., Ed.; Academic Press, 1971; pp 15-73.

2. Bauer, E. J. Phys.: Condens. Matter 2009, 21, 314001. doi:10.1088/0953-8984/21/31/314001

3. Bauer, E. J. Electron Spectrosc. Relat. Phenom. 2012, 185, 314-322. doi:10.1016/j.elspec.2012.08.001

4. Tonner, B. P.; Harp, G. R. Rev. Sci. Instrum. 1988, 59, 853. doi:10.1063/1.1139792

5. Locatelli, A.; Bauer, E. J. Phys.: Condens. Matter 2008, 20, 093002. doi:10.1088/0953-8984/20/9/093002

6. Bauer, E. Rep. Prog. Phys. 1994, 57, 895. doi:10.1088/0034-4885/57/9/002

7. Man, K. L.; Altman, M. S. J. Phys.: Condens. Matter 2012, 24, 314209. doi:10.1088/0953-8984/24/31/314209

8. Wintterlin, J.; Bocquet, M.-L. Surf. Sci. 2009, 603, 1841-1852. doi:10.1016/j.susc.2008.08.037

9. Batzill, M. Surf. Sci. Rep. 2012, 67, 83-115. doi:10.1016/j.surfrep.2011.12.001

10. Tetlow, H.; Posthuma de Boer, J.; Ford, I. J.; Vvedensky, D. D.; Coraux, J.; Kantorovich, L. Phys. Rep. 2014, 542, 195-295. doi:10.1016/j.physrep.2014.03.003

11. Altman, M. S. J. Phys.: Condens. Matter 2010, 22, 084017. doi:10.1088/0953-8984/22/8/084017

12. Telieps, W.; Bauer, E. Ultramicroscopy 1985, 17, 57-65. doi:10.1016/0304-3991(85)90177-9

13. Bauer, E. Surf. Rev. Lett. 1998, 5, 1275. doi:10.1142/S0218625X98001614

14. Tanuma, S.; Powell, C. J.; Penn, D. R. Surf. Interface Anal. 2011, 43, 689-713. doi:10.1002/sia.3522

15. Altman, M. S.; Chung, W. F.; He, Z. Q.; Poon, H. C.; Tong, S. Y. Appl. Surf. Sci. 2001, 169-170, 82-87. doi:10.1016/S0169-4332(00)00644-9

16. Zdyb, R.; Menteş, T. O.; Locatelli, A.; Niño, M. A.; Bauer, E. Phys. Rev. B 2013, 87, 075436. doi:10.1103/PhysRevB.87.075436

17. Reichelt, R.; Günther, S.; Wintterlin, J.; Moritz, W.; Aballe, L.; Menteş, T. O. J. Chem. Phys. 2007, 127, 134706. doi:10.1063/1.2779028

18. de Groot, F. M. F. J. Electron Spectrosc. Relat. Phenom. 1994, 67, 529-622. doi:10.1016/0368-2048(93)02041-J

19. Hannon, J. P.; Trammell, G. T.; Blume, M.; Gibbs, D. Phys. Rev. Lett. 1988, 61, 1245. doi:10.1103/PhysRevLett.61.1245

20. Schneider, C. M.; Schönhense, G. Rep. Prog. Phys. 2002, 65, 1785. doi:10.1088/0034-4885/65/12/202

21. Hüfner, S. Photoelectron Spectroscopy; Springer-Verlag: Berlin Heidelberg New York, 2003.

22. Menteş, T. O.; Locatelli, A. J. Electron Spectrosc. Relat. Phenom. 2012, 185, 323-329. doi:10.1016/j.elspec.2012.07.007

23. Barrett, N.; Conrad, E.; Winkler, K.; Krömker, B. Rev. Sci. Instrum. 2012, 83, 083706. doi:10.1063/1.4746279

24. Locatelli, A.; Aballe, L.; Menteş, T. O.; Kiskinova, M.; Bauer, E. Surf. Interface Anal. 2006, 38, 1554-1557. doi:10.1002/sia.2424

25. Schmidt, T.; Heun, S.; Slezak, J.; Diaz, J.; Prince, K. C.; Lilienkamp, G.; Bauer, E. Surf. Rev. Lett. 1998, 5, 1287. doi:10.1142/S0218625X98001626
26. Gadzuk, W.; Plummer, E. W. Rev. Mod. Phys. 1973, 45, 487. doi:10.1103/RevModPhys.45.487

27. Tosi, L.; Diviacco, B.; lazzourene, F.; Roux, R.; Walker, R. P.; Zangrando, D. Commissioning of elliptical undulators in Elettra. In Proceedings of EPAC 2000, 2000; pp $2349 \mathrm{ff}$.

28. Locatelli, A.; Bianco, A.; Cocco, D.; Cherifi, S.; Heun, S.; Marsi, M.; Pasqualetto, M.; Bauer, E. J. Phys. IV 2003, 104, 99-102. doi:10.1051/jp4:200300038

29. Locatelli, A.; Menteş, T. O.; Niño, M. A.; Bauer, E. Ultramicroscopy 2011, 111, 1447-1454. doi:10.1016/j.ultramic.2010.12.020

30. Cocco, D.; Marsi, M.; Kiskinova, M.; Prince, K. C.; Schmidt, T.; Heun, S.; Bauer, E. Proc. SPIE 1999, 3767, 271. doi:10.1117/12.371126

31. Imhof, R. E.; Adams, A.; King, G. C. J. Phys. E: Sci. Instrum. 1976, 9 , 138. doi:10.1088/0022-3735/9/2/024

32. The Doniach-Šunjić lineshape for the bulk/surface components were fitted with 60/129 meV Lorentzian broadening and 0.035/0.063 asymmetry, respectively. The surface component was found to be at 320 meV lower binding energy.

33. Menteş, T. O.; Niño, M. A.; Locatelli, A. e-J. Surf. Sci. Nanotechnol. 2011, 9, 72-79. doi:10.1380/ejssnt.2011.72

34. Schmidt, T.; Sala, A.; Marchetto, H.; Umbach, E.; Freund, H.-J. Ultramicroscopy 2013, 126, 23-32. doi:10.1016/j.ultramic.2012.11.004

35. Sutter, P. W.; Flege, J.-I.; Sutter, E. A. Nat. Mater. 2008, 7, 406-411. doi:10.1038/nmat2166

36. Loginova, E.; Bartelt, N. C.; Feibelman, P. J.; McCarty, K. F. New J. Phys. 2008, 10, 093026. doi:10.1088/1367-2630/10/9/093026

37. Loginova, E.; Bartelt, N. C.; Feibelman, P. J.; McCarty, K. F. New J. Phys. 2009, 11, 063046. doi:10.1088/1367-2630/11/6/063046

38. McCarty, K. F.; Feibelman, P. J.; Loginova, E.; Bartelt, N. C. Carbon 2009, 47, 1806-1813. doi:10.1016/j.carbon.2009.03.004

39. Sutter, P.; Sadowski, J. T.; Sutter, E. A. J. Am. Chem. Soc. 2010, 132, 8175-8179. doi:10.1021/ja102398n

40. Sutter, P.; Albrecht, P.; Tong, X.; Sutter, E. J. Phys. Chem. C 2013, 117, 6320-6324. doi:10.1021/jp400838j

41. Alfé, D.; Pozzo, M.; Miniussi, E.; Günther, S.; Lacovig, P.; Lizzit, S.; Larciprete, R.; Burgos, B. S.; Menteş, T. O.; Locatelli, A.; Baraldi, A. Sci. Rep. 2013, 3, No. 2430. doi:10.1038/srep02430

42. Feenstra, R. M.; Srivastava, N.; Gao, Q.; Widom, M.; Diaconescu, B.; Ohta, T.; Kellogg, G. L.; Robinson, J. T.; Vlassiouk, I. V. Phys. Rev. B 2013, 87, 041406. doi:10.1103/PhysRevB.87.041406

43. Locatelli, A.; Knox, K. R.; Cvetko, D.; Menteş, T. O.; Niño, M. Á.; Wang, S.; Yilmaz, M. B.; Kim, P.; Osgood, R. M., Jr.; Morgante, A. ACS Nano 2010, 4, 4879-4889. doi:10.1021/nn101116n

44. Miniussi, E.; Pozzo, M.; Baraldi, A.; Vesselli, E.; Zhan, R. R.; Comelli, G.; Menteş, T. O.; Niño, M. A.; Locatelli, A.; Lizzit, S.; Alfé, D. Phys. Rev. Lett. 2011, 106, 216101. doi:10.1103/PhysRevLett.106.216101

45. Miniussi, E.; Pozzo, M.; Menteş, T. O.; Niño, M. A.; Locatelli, A.; Vesselli, E.; Comelli, G.; Lizzit, S.; Alfè, D.; Baraldi, A. Carbon 2014, 73, 389-402. doi:10.1016/j.carbon.2014.02.081

46. Kraus, J.; Böcklein, S.; Reichelt, R.; Günther, S.; Santos, B.; Menteş, T. O.; Locatelli, A. Carbon 2013, 64, 377-390. doi:10.1016/j.carbon.2013.07.090

47. Wofford, J. M.; Nie, S.; McCarty, K. F.; Bartelt, N. C.; Dubon, O. D. Nano Lett. 2010, 10, 4890-4896. doi:10.1021/nl102788f

48. Walter, A. L.; Nie, S.; Bostwick, A.; Kim, K. S.; Moreschini, L.; Chang, Y. J.; Innocenti, D.; Horn, K.; McCarty, K. F.; Rotenberg, E. Phys. Rev. B 2011, 84, 195443. doi:10.1103/PhysRevB.84.195443 
49. Sun, J.; Hannon, J. B.; Tromp, R. M.; Johari, P.; Bol, A. A.; Shenoy, V. B.; Pohl, K. ACS Nano 2010, 4, 7073-7077. doi:10.1021/nn102167f

50. Locatelli, A.; Wang, C.; Africh, C.; Stojić, N.; Menteş, T. O.; Comelli, G.; Binggeli, N. ACS Nano 2013, 7, 6955-6963. doi:10.1021/nn402178u

51. Locatelli, A.; Zamborlini, G.; Menteş, T. O. Carbon 2014, 74, 237-248. doi:10.1016/j.carbon.2014.03.028

52. Vinogradov, N. A.; Zakharov, A. A.; Kocevski, V.; Rusz, J.; Simonov, K. A.; Eriksson, O.; Mikkelsen, A.; Lundgren, E.; Vinogradov, A. S.; Mårtensson, N.; Preobrajenski, A. B. Phys. Rev. Lett. 2012, 109, 026101. doi:10.1103/PhysRevLett.109.026101

53. Knox, K. R.; Wang, S.; Morgante, A.; Cvetko, D.; Locatelli, A.; Menteş, T. O.; Niño, M. Á.; Kim, P.; Osgood, R. M., Jr. Phys. Rev. B 2008, 78, 201408. doi:10.1103/PhysRevB.78.201408

54. Knox, K. R.; Locatelli, A.; Yilmaz, M. B.; Cvetko, D.; Menteş, T. O.; Niño, M. Á.; Kim, P.; Morgante, A.; Osgood, R. M., Jr. Phys. Rev. B 2011, 84, 115401. doi:10.1103/PhysRevB.84.115401

55. Sutter, P.; Hybertsen, M. S.; Sadowski, J. T.; Sutter, E. Nano Lett. 2009, 9, 2654-2660. doi:10.1021/n1901040v

56. Sutter, P.; Sadowski, J. T.; Sutter, E. Phys. Rev. B 2009, 80, 245411. doi:10.1103/PhysRevB.80.245411

57. Zakharov, A. A.; Mikkelsen, A.; Andersen, J. N. J. Electron Spectrosc. Relat. Phenom. 2012, 185, 417-428. doi:10.1016/j.elspec.2012.03.002

58. Pacilé, D.; Papagno, M.; Rodríguez, A. F.; Grioni, M.; Papagno, L.; Girit, Ç. Ö.; Meyer, J. C.; Begtrup, G. E.; Zettl, A. Phys. Rev. Lett. 2008, 101, 066806. doi:10.1103/PhysRevLett.101.066806

59. Johnson, K.; Ge, Q.; Sauerhammer, B.; Titmuss, S.; King, D. Surf. Sci. 2001, 478, 49-56. doi:10.1016/S0039-6028(00)01092-X

60. Locatelli, A.; Sbraccia, C.; Heun, S.; Baroni, S.; Kiskinova, M. J. Am. Chem. Soc. 2005, 127, 2351-2357. doi:10.1021/ja045285k

61. N'Diaye, A. T.; van Gastel, R.; Martínez-Galera, A. J.; Coraux, J.; Hattab, H.; Wall, D.; Meyer zu Heringdorf, F.-J.; Horn-von Hoegen, M.; Gómez-Rodríguez, J. M.; Poelsema, B.; Busse, C.; Michely, T. New J. Phys. 2009, 11, 113056. doi:10.1088/1367-2630/11/11/113056

62. Hattab, H.; N'Diaye, A. T.; Wall, D.; Klein, C.; Jnawali, G.; Coraux, J.; Busse, C.; van Gastel, R.; Poelsema, B.; Michely, T.;

Meyer zu Heringdorf, F.-J.; Horn-von Hoegen, M. Nano Lett. 2012, 12, 678-682. doi:10.1021/nl203530t

63. Preobrajenski, A. B.; Ng, M. L.; Vinogradov, A. S.; Mårtensson, N. Phys. Rev. B 2008, 78, 073401. doi:10.1103/PhysRevB.78.073401

64. Varykhalov, A.; Sánchez-Barriga, J.; Shikin, A. M.; Biswas, C.; Vescovo, E.; Rybkin, A.; Marchenko, D.; Rader, O. Phys. Rev. Lett. 2008, 101, 157601. doi:10.1103/PhysRevLett.101.157601

65. Khomyakov, P. A.; Giovannetti, G.; Rusu, P. C.; Brocks, G.; van den Brink, J.; Kelly, P. J. Phys. Rev. B 2009, 79, 195425. doi:10.1103/PhysRevB.79.195425

66. Giovannetti, G.; Khomyakov, P. A.; Brocks, G.; Karpan, V. M.; van den Brink, J.; Kelly, P. J. Phys. Rev. Lett. 2008, 101, 026803. doi:10.1103/PhysRevLett.101.026803

67. Alerhand, O. L.; Vanderbilt, D.; Meade, R. D.; Joannopoulos, J. D. Phys. Rev. Lett. 1988, 61, 1973. doi:10.1103/PhysRevLett.61.1973

68. Menteş, T. O.; Locatelli, A.; Aballe, L.; Bauer, E. Phys. Rev. Lett. 2008, 101, 085701. doi:10.1103/PhysRevLett.101.085701

69. Menteş, T. O.; Stojić, N.; Locatelli, A.; Aballe, L.; Binggeli, N.; Niño, M. A.; Kiskinova, M.; Bauer, E. EPL 2011, 94, 38003. doi:10.1209/0295-5075/94/38003

70. Menteş, T. O.; Locatelli, A.; Aballe, L.; Niño, M. A.; Bauer, E. Ultramicroscopy 2013, 130, 82-86. doi:10.1016/j.ultramic.2013.03.012
71. Monti, M.; Santos, B.; Mascaraque, A.; Rodríguez de la Fuente, O.; Niño, M. A.; Menteş, T. O.; Locatelli, A.; McCarty, K. F.; Marco, J. F.; de la Figuera, J. J. Phys. Chem. C 2012, 116, 11539-11547. doi:10.1021/jp300702d

72. Monti, M.; Santos, B.; Mascaraque, A.; Rodríguez de la Fuente, O.; Niño, M. A.; Menteş, T. O.; Locatelli, A.; McCarty, K. F.; Marco, J. F.; de la Figuera, J. Phys. Rev. B 2012, 85, 020404(R). doi:10.1103/PhysRevB.85.020404

73. Sala, A.; Marchetto, H.; Qin, Z.-H.; Shaikhutdinov, S.; Schmidt, T.; Freund, H.-J. Phys. Rev. B 2012, 86, 155430. doi:10.1103/PhysRevB.86.155430

74. Noguera, C. J. Phys.: Condens. Matter 2010, 12, R367. doi:10.1088/0953-8984/12/31/201

75. Genuzio, F.; Sala, A.; Schmidt, T.; Menzel, D.; Freund, H.-J. J. Phys. Chem. C, in press. doi:10.1021/jp504020a

\section{License and Terms}

This is an Open Access article under the terms of the Creative Commons Attribution License (http://creativecommons.org/licenses/by/2.0), which permits unrestricted use, distribution, and reproduction in any medium, provided the original work is properly cited.

The license is subject to the Beilstein Journal of Nanotechnology terms and conditions: (http://www.beilstein-journals.org/bjnano)

The definitive version of this article is the electronic one which can be found at: doi:10.3762/bjnano.5.198 